\title{
1 Variability in fitness effects and the limitations of fitness optimization
}

2

3 Christopher J Graves ${ }^{1 *}$ and Daniel M Weinreich ${ }^{1 *}$

4

$5{ }^{1}$ Brown University, Department of Ecology and Evolutionary Biology and Center for

6 Computational and Molecular Biology. Providence, RI, USA

7

8 * Corresponding authors: christopher_graves@brown.edu and daniel_weinreich@brown.edu;

9 Box G-W, 80 Waterman Street

10 Brown University, Providence, RI 02912. Phone: 401-863-3937.

11

12 Running title: Limitations of fitness optimization

13

14 Keywords: Natural selection, Fitness optimization, Varying environments, Group selection,

15 Inclusive fitness, Mutation rate

16 


\section{Abstract}

19 Evolutionary biologists commonly assess the evolutionary advantage of an allele based on its

20 effects on the lifetime survival and reproduction of individuals. However, alleles affecting traits

21 like sex, evolvability, and cooperation can cause fitness effects that depend heavily on

22 differences in the environmental, genetic, and social context of individuals carrying the allele.

23 This variability makes it difficult to summarize the evolutionary fate of an allele based solely on

24 its effects on any one individual. In this review we show how attempts to average over variability

25 in the fitness effects of an allele can sometimes cause misleading results. We then describe a

26 number of intriguing new evolutionary phenomena that have emerged in studies that explicitly

27 model the fate of alleles that influence long-term lineage dynamics. We conclude with prospects

28 for generalizations of population genetics theory and discuss how this theory might be applied to

29 the evolution of infectious diseases. 


\section{Contents}

\section{I. INTRODUCTION}

\section{II. LINEAGE-INVARIANT FITNESS EFFECTS}

34 III. LINEAGE-VARIABLE FITNESS EFFECTS

$35 \quad$ Environmental Interactions

$36 \quad$ Genetic Interactions

$37 \quad$ Social Interactions

38 IV. LIMITATIONS TO FITNESS AVERAGES

39 Limitations due to short-sighted selection

$40 \quad$ Beyond fitness averages

41 V. IMPLICATIONS FOR INFECTIOUS DISEASE EVOLUTION

42 VI. CONCLUSIONS

43 


\section{Introduction}

46 Evolution by natural selection is driven by heritable differences in the reproductive success of

47 individuals. However, the long-term outcome of natural selection depends not only on the effects

48 of an allele on individual bearers but also on its effects across its entire lineage of descendants-

49 defined here as the genealogy of an allele from its origination to its ultimate fixation or

50 extinction in the population (Sidebar 1). When fitness effects are invariant across a lineage, the

51 long-term fate of an allele can be deduced in a relatively straightforward manner from its

52 recursive effects on survival and reproduction across descendent members of the lineage. In

53 other cases, the evolutionary success of an allele is not an obvious consequence of its effects on

54 individuals. For example, variable environments can cause the same allele to have differing

55 effects on fitness depending on an individuals' environmental context. Similarly, fitness effects

56 may vary due to the presence of other alleles in the genome, which are themselves polymorphic

57 in the population. In such cases, it is often presumed that traits will tend to spread by natural

58 selection so long as they are beneficial to their carriers on average (Eshel 1973, Nunney 1999).

59 This implies that natural selection favors traits that are beneficial not strictly to individuals, but

60 to genetic lineages as a whole.

61

62 The concept that natural selection may optimize quantities related to the average success of an

63 allele across a lineage has arisen in a wide range of problems ranging from varying environments

64 to the evolution of sex and cooperation (Akçay and Van Cleve 2016, Eshel 1973, Kussell and

65 Leibler 2005, Lehmann, et al. 2016, Nunney 1999, Nunney 1999). In general, this idea arises

66 when the fitness effect of an allele varies between individual carriers, thereby limiting the ability 
67 to infer the long-term success of an allele based on measures of individual fitness alone. A large

68 class of evolutionary problems fit this description and they can be classified by whether the

69 variability across a lineage arises due to environmental, genetic, or social factors. We outline

70 examples of each in Table 1 and describe them in more detail in the main text. Each source of

71 variation has largely been discussed within its own body of literature, where equivalent concepts

72 are used to describe a distinct set of adaptations, often with distinct terminology. Despite some

73 obvious similarities, there have been few attempts at synthesizing what is known in each of these

74 cases into a formal quantitative theory of the evolution of alleles with lineage-variable fitness

75 effects.

77 Averaging the fitness effects of an allele across a lineage shifts the target of adaptation from

78 individuals to lineages. However, one must acknowledge possible limitations in the ability of

79 natural selection to favor traits that confer a long-term benefit to a lineage. Specifically, natural

80 selection is myopic in nature- acting to increase the frequency of traits that confer an immediate

81 advantage to individuals without regard to their future utility to a lineage. This shortsightedness

82 can have dramatic consequences, particularly if it results in the permanent extinction of an allele

83 prior to it realizing any average benefit in the long-term. Indeed, the notion that natural selection

84 will act most strongly on alleles that confer a short-term advantage was championed by Maynard

85 Smith (1964) and Williams (1966) in their now famous critique of group selection, and is still in

86 use (Lynch 2007, Sniegowski and Murphy 2006). When does natural selection favor traits that

87 confer a long-term benefit averaged across a lineage and when does shortsighted selection limit

88 this ability? 
90 After briefly summarizing results from classical, lineage-invariant theory that successfully

91 relates individual fitness to a lineage's eventual fate, we discuss a diversity of examples of

92 lineage-variable fitness, i.e., cases in which the fitness effects of an allele vary across its lineage

93 of descendants. We illustrate the shortcomings of averaging variability across the lineage in

94 finite populations, in which alleles that are beneficial in the long-term are nevertheless

95 vulnerable to extinction. Consequently, shortsighted selection in finite populations can limit the

96 ability of natural selection to optimize even these measures of fitness. Finally, we discuss other

97 counterintuitive results that emerge in examples where lineage-variable fitness is modeled

98 explicitly. These results show that the fate of an allele can be sensitive not only to its fitness

99 effects across a lineage, but to features unrelated to classical notions of fitness, such as the

100 population size. We conclude by highlighting implications for the evolution of infectious

101 diseases and directions for future work.

102

\section{Lineage-Invariant Fitness Effects}

105 Evolutionary biologists are fundamentally concerned with understanding the outcome of natural selection on traits that influence the survival and reproduction of their carriers. Before discussing

107 cases in which the fitness effects of an allele are variable across its lineage we first consider the 108 case where fitness effects are invariant across a lineage. Our approach throughout will be on the

109 field of population genetics, which has a rich tradition of analyzing dynamical models that

110 combine various evolutionary forces including natural selection, genetic drift, and mutation.

111 Such dynamical treatments of evolution provide a comprehensive analysis of a lineage - starting

112 from its origination in the population and ending with its ultimate fixation or extinction. We will 
113 therefore be decidedly brief in our overview of other aspects of evolutionary theory, which

114 include techniques such as game theory and quantitative genetics.

116 Consider an allele that influences the expected number of surviving offspring produced over the

117 lifetime of its carriers. Formally, we allow the precise number of offspring produced by any

118 particular individual in this lineage to be a Poisson random variable drawn independently from

119 an identical distribution, with mean defined as the Wrightian fitness, $w$. This concept of fitness

120 articulates well with the Darwinian notion of fitness as lifetime reproductive success. The most

121 fundamental consideration regarding the fate of an allele by natural selection is whether the allele

122 influences this measure of fitness relative to the resident "wild type" in the population. In

123 population genetics, this fitness effect is most often denoted with the selection coefficient, $s$,

124 defined as the proportional change in expected number of offspring relative to the wild-type: $s \equiv$

$125 w_{\mathrm{mut}} / w_{\mathrm{wt}}-1$.

127 Now consider a population with constant size, $N$. Since the number of surviving offspring born

128 to an individual is a random variable, we allow for random fluctuations in the number of

129 individuals carrying an allele as the basis for genetic drift. Assuming that generations are discrete

130 and non-overlapping and approximating the Poisson offspring distribution with a binomial so

131 that the population size remains fixed, we can describe the allele frequency dynamics using the

132 Wright-Fisher model. We emphasize that the Wright-Fisher process and related models capture

133 the interplay between natural selection and genetic drift in finite populations by incorporating

134 stochasticity in the number of surviving offspring born to each individual. However, by 
135 definition all models of lineage-invariant selection assume that the distribution in that number

136 remains constant across the lineage (Figure 1A).

138 Given this framework, we can obtain solutions for a number of quantities pertaining to the fate of 139 a mutant allele based on its selection coefficient, $s$. Of particular interest given our concern with

140 the ultimate fate of a lineage is the probability that an allele eventually displaces all alternatives

141 in the population, known as the probability of fixation, $P_{\text {fix }}$ Kimura (Kimura 1962) found this

142 quantity for a mutation starting at frequency $x_{0}$, in a haploid, randomly mating population of size

$143 N$, using a continuous diffusion approximation of the Wright-Fisher process:

$$
P_{\text {fix }}\left(N, s, x_{0}\right)=\frac{1-\mathrm{e}^{-N s x_{0}}}{1-\mathrm{e}^{-N s}}
$$

145 This result highlights many of the key features of classical population genetics theory. Solving

146 for the limit as $s$ approaches zero leads to $P_{\mathrm{fix}}=x_{0}$. This defines the neutral expectation that the 147 probability of fixation of an allele is simply equal to its starting frequency. Focusing on the case

148 where an allele starts from a single mutation in the population, we take $x_{0}=1 / N$. Now consider a

149 beneficial mutation, $s>0$. Here, $P_{\text {fix }}>1 / N$, but only asymptotically approaches $s$, even as

150 population size $N$ tends to infinity (Haldane 1927). In other words, fixation of even a strongly

151 beneficial mutation is not assured, reflecting the fact genetic drift dominates allele frequency

152 dynamics until there are roughly $1 / s$ copies in the population. This effect is worsened in small

153 populations since $1 / s$ copies may be an appreciable fraction of the population. Thus as $s$ or $N$ get 154 small, $1 / s$ approaches $N$ and genetic drift comes to dominate selection. This result implies that 155 mutations are effectively neutral from the standpoint of natural selection, unless $s>1 / N$. Finally, 156 and somewhat less intuitively, Kimura's formula also shows that even deleterious mutations $(s<$ 
157 0), can have a nonzero fixation probability. Here again, genetic drift can overwhelm natural 158 selection in populations roughly no larger than $1 /|S|$ individuals.

\section{Lineage-Variable Fitness Effects}

162 Under the assumption that an allele exerts a constant, lineage-invariant effect on fitness,

163 Equation 1 demonstrates that a mutant's fitness effect is sufficient to predict the fate of its

164 lineage. We now turn to cases where variability in the fitness effects of an allele can cause this

165 result to fail. Examples of lineage-variable fitness effects emerge under many realistic biological

166 scenarios, where alleles do not act alone to influence fitness but interact with different

167 environmental, genetic, or social factors (Table 1, Figure 1). Consequently, the number of

168 offspring produced by individuals in a lineage may not be drawn from any fixed distribution,

169 violating the assumption of lineage invariance underlying Equation 1. We emphasize that such

170 variability in offspring number is beyond that captured in models like Wright-Fisher, which

171 typically require the distribution of offspring number to be fixed. Our goal in this section is to

172 highlight some of the relevant examples of variability in fitness of an allele represented by the

173 three classes in Table 1, and to build some intuition for how they have been handled in the

174 literature. We also seek to show that adaptations associated with each example depend uniquely

175 on the effects of an allele on the fate of a lineage rather than on individual success.

Environmental interactions

179 Natural environments are inherently variable and therefore present an obvious challenge to the assumption that an allele will have the same effect on fitness for all members of a lineage. 
181 Variation in the environment over time will cause contemporary members of a lineage to

182 experience the same distribution of offspring number, but this distribution now depends on time

183 (Figure 1B). Contrastingly, under spatial variation in the environment, contemporary members

184 will experience fitness effects that depend on the interaction between their shared allele and the

185 local environment they encounter. This again implies that no single distribution in offspring

186 number will be generally applicable. In either case, if environmental change is so rapid that

187 individuals encounter a succession of different environments in their lifetime, then fitness can be

188 described as a lifetime average of total survival and reproduction (Levins 1968). We will

189 therefore focus on the more interesting case where environments vary on a timescale greater than

190 the generation time of the organism; here averaging can often be misleading.

192 The greatest progress has been made in models of temporally varying environments, in which

193 case the selection coefficient $s$ is no longer a constant, but a time-dependent quantity, $s(t)$.

194 Formal analysis typically requires specifying a particular form of $s(t)$ at the expense of

195 generality. It is commonly assumed that environments are randomly drawn from a fixed

196 distribution or that that the population size is infinite (Dempster 1955, Gillespie 1973, Kussell

197 and Leibler 2005, Lewontin and Cohen 1969). Under these assumptions, a diverse set of models

198 can be integrated based on how variation in fitness correlates within and between members of

199 two competing lineages (Frank and Slatkin 1990). We note, however, that such an approach is

200 limited to deriving the instantaneous change in allele frequency rather than explicitly modeling

201 lineage dynamics. Another consequence of assuming random environmental change and infinite

202 populations is that natural selection will favor alleles that increase the long-term growth rate of a

203 lineage, averaged over all environments (Dempster 1955, Gillespie 1973, Kussell and Leibler 
2005, Lewontin and Cohen 1969, Stearns 2000). Formally, this corresponds to an increase in the

205 geometric mean fitness, or equivalently, the mean intrinsic growth rate (Sidebar 2), and is

206 generalizable to other forms of $s(t)$ (Cvijović, et al. 2015). Importantly, even arbitrarily large but

207 finite populations experience genetic drift, which can limit the ability to maximize the long-term

208 growth rate of lineages in certain environmental scenarios. We discuss these limitations in more

209 detail below.

210

211 Despite its limitations in finite populations, the principle that in variable environments natural

212 selection acts to increase geometric mean fitness is a key theoretical insight, and it is presumed

213 to underlie numerous adaptations. These include strategies like developmental and phenotypic

214 plasticity that allow adaptive phenotypic responses to environmental conditions that may not be

215 encountered by all individuals (Meyers and Bull 2002, Via, et al. 1995). Most notable is the

216 evolution of bet-hedging traits in which an allele causes the exaggeration of phenotypic noise

217 among members of a lineage, thereby allowing a single genotype to spread environmental risk

218 among different phenotypes that are suited to different environments (Fraser and Kaern 2009,

219 Gillespie 1974, Kussell and Leibler 2005, Philippi and Seger 1989). Such a strategy is inherently

220 dependent on selection favoring traits that confer a long-term benefit across a lineage, since

221 individuals will experience differing fitness values depending on their phenotype and the

222 environment they encounter. By spreading the risk of fitness losses under future environmental

223 uncertainty across members of a lineage, bet-hedging helps to ensure survival and reproduction

224 across the lineage as a whole, regardless of the environment. Examples of adaptive bet-hedging

225 strategies have been noted in plants (Childs, et al. 2010, Clauss and Venable 2000, Gremer and 
226 Venable 2014), insects (Hopper 1999, Menu, et al. 2000), and microbes (Balaban, et al. 2004,

227 Jones and Lennon 2010, Levy, et al. 2012).

228

229 Genetic interactions

230

231 Alleles don't influence fitness alone but do so as part of an integrated genome. The genetic

232 background of an allele is therefore another important source of variability in fitness across a

233 lineage. Perhaps the most obvious example is that of epistasis (Phillips 1998), in which the

234 fitness effect of a mutation depends on its genetic context. Empirical evidence suggests that

235 epistasis among alleles is widespread (Costanzo, et al. 2016, Kryazhimskiy, et al. 2014, Wang, et

236 al. 2014, Weinreich, et al. 2013) and therefore provides an important source of variability in the

237 fitness effects of an allele, particularly in sexual populations. Similar variation in fitness can

238 occur in asexual populations due to secondary mutations that arise on the genetic background of

239 an allele as it spreads. This effect is most important in large populations or under high mutation

240 rates. Such conditions lead to clonal interference (Gerrish and Lenski 1998), in which multiple

241 asexual lineages carry competing beneficial mutations, thereby interfering with one another's

242 fixation. The fate of a lineage under clonal interference cannot be decided by the selection

243 coefficient of a single allele, but instead depends on the process of successive mutations

244 accumulating along a series of competing asexual lineages (Desai and Fisher 2007, Lang, et al.

245 2013). Indeed, this presents a major hurdle to evolving asexual populations, since the lack of

246 recombination implies strict genetic linkage among mutations that occur on the same

247 background. This lack of recombination can also lead to Muller's ratchet (Haigh 1978, Muller 
248 1964), in which the serial fixation of deleterious mutations by genetic drift can cause fitness to

249 erode along an asexual lineage.

250

251 The constraints on asexual adaptation due to clonal interference and Muller's ratchet provide

252 strong arguments for why so many organismal life cycles include periods of recombination or

253 sexual reproduction. These arguments are invariably related to the idea that alleles influencing

254 sex can increase the long-term average evolutionary success of a lineage (Nunney 1999). This is

255 because sex is inherently costly to individuals, who must invest time and energy in mating and

256 further invest resources into the production of males, which are not capable of independent

257 reproduction (Maynard Smith 1978). These costs could, however, be balanced if sex increases

258 the long-term fitness of lineages (Nunney 1989, Nunney 1999). For example, under certain

259 conditions of epistasis, recombination can accelerate both the pace of adaptation (Eshel and

260 Feldman 1970) and the ability of populations to purge deleterious mutations and fend off

261 Muller's ratchet (Kondrashov 1988). Furthermore, sexual reproduction can increase rates of

262 adaptation by allowing beneficial mutations that arise on different backgrounds to be combined

263 into a single genotype, thereby limiting the constraints imposed by clonal interference (Cooper

264 2007, McDonald, et al. 2016). Finally, the red-queen hypothesis (Hamilton, et al. 1990, Van

265 Valen 1973), asserts that the constant creation of new genotypes under recombination can allow

266 organisms to more readily compete in a co-evolutionary arms race with parasites. Indeed, sex is

267 likely to have evolved for a combination of reasons and empirical observations support many of

268 the hypotheses that have been put forth (Colegrave 2002, Cooper 2007, Goddard, et al. 2005,

269 McDonald, et al. 2016, Morran, et al. 2011). 
271 Sex and recombination are not the only processes that increase rates of adaptation. There has

272 been substantial recent attention on whether natural selection can act more generally on the

273 ability of populations to adapt, or its evolvability. Selection for evolvability is contentious, since

274 the ability to adapt to future contingencies is a feature of populations and would therefore appear

275 to require evolutionary foresight and group selection operating on biological populations (Lynch

276 2007, Pigliucci 2008, Sniegowski and Murphy 2006, but see Watson and Szathmáry 2016).

277 However, traits that increase evolvability could also arise by the process of natural selection

278 favoring traits that are beneficial on average, with lineages being more likely to persist over

279 longer evolutionary periods if they are able to adapt to future conditions (Eshel 1973). While

280 numerous traits could increase evolvability (Wagner and Altenberg 1996), there has been a great

281 deal of attention paid to the evolution of alleles which influence the mutation rate - known as

282 mutation rate modifiers (Denamur and Matic 2006, Sniegowski, et al. 2000). Mutation rate

283 modifiers have been observed in microbial populations both in the lab (Sniegowski, et al. 1997)

284 and in nature (LeClerc, et al. 1996, Matic, et al. 1997). The fate of such "mutator" alleles is

285 intriguing, since they often arise without a direct effect on fitness themselves (Chao and Cox

286 1983, Sniegowski, et al. 1997). In asexual populations, mutators are still physically linked to the

287 mutations they produce and can thereby influence the statistical properties and long-term fate of

288 lineages (Figure 1C). In such scenarios, evolvability arises as a by-product of indirect selection

289 and genetic hitchhiking of mutators (Sniegowski and Murphy 2006). However, there are also

290 notable exceptions in which histories of repeated environmental change could directly favor the

291 evolution of traits that increase evolvability. This appears to be the case in pathogens, where

292 elevated mutation rates in antigens to increase the capacity to adapt to a dynamic vertebrate

293 immune response (Graves, et al. 2013, Moxon, et al. 1994). 
Social interactions

297 Fitness is influenced not only by environmental and genetic factors but also by interactions with

298 other conspecifics. These interactions can create a type of lineage-variable fitness known as

299 frequency dependent selection, where the fitness effects of an allele are dependent on the

300 frequency of the allele in the population. Frequency dependence is conveniently analyzed in the

301 context of evolutionary game theory (Sidebar 3), which allows one to consider the ability of an

302 initially rare allele to invade a population fixed for a wild-type allele (Maynard Smith 1982,

303 Maynard Smith and Price 1973). This approach provides a generalization of the concept of a

304 selection coefficient to instances where fitness cannot be wholly represented by a constant value.

305 A classic example of frequency-dependent selection arises when considering cooperative traits.

306 Here, cooperative acts incur a cost to individuals and are therefore susceptible to invasion by

307 selfish "cheater" strategies that avoid the cost of cooperating while still reaping the benefit.

308 Cheaters are typically beneficial when rare, since their fitness advantage requires interactions

309 with other cooperators. Despite the inherent susceptibility to cheaters, cooperation is common in

310 nature and is presumed to underlie major transitions in evolutionary history, such as the

311 evolution of multicellularity (Szathmary and Maynard Smith 1995). The mechanisms promoting

312 the evolution and maintenance of cooperation are therefore of long-standing interest to

313 biologists.

315 Significant theoretical progress on the evolution of cooperation arose with the formulation of 316 inclusive fitness theory. Hamilton (1964) showed that genes controlling cooperation may be 
317 beneficial on average so long as the beneficiary of cooperative actions are kin, which are likely

318 to share the genes controlling cooperation by common descent. The key realization of this theory

319 is that cooperative acts need not directly increase the reproductive success of individual bearers,

320 but instead must increase the average effect of a gene across the lineage of cooperators (Akçay

321 and Van Cleve 2016). Cooperation can also be stable under cases of multi-level selection (Luo

322 2014, Simon, et al. 2013, Traulsen and Nowak 2006). The formation and dissolution of new

323 groups is itself a reproductive process and the long-term fate of a lineage is therefore sensitive to

324 the influence of an allele on group-level reproduction (Figure 1D). A well-known example is

325 infectious diseases, discussed below, in which individual cells or viral particles must replicate

326 within hosts and also spread among hosts to establish new infections.

328 There is ample empirical evidence for the stability of cooperative traits in nature if selection

329 favors traits that increase the long-term growth rate of a lineage at the expense of individual

330 fitness. For example, a large number of studies have shown how cooperation, which appears

331 costly to individuals, can prevail through the action of group selection and kin selection (Gore, et

332 al. 2009, Koschwanez, et al. 2013, Rainey and Rainey 2003, Turner and Chao 1999, Velicer, et

333 al. 2000). Perhaps more intriguing is the evolution of "policing" phenotypes that function to

334 reduce the short-term benefits of selfish cheater phenotypes and thereby stabilize cooperation

335 (Frank 1995, Nunney 1999, Travisano and Velicer 2004). For example, in social insects,

336 reproduction by the worker caste constitutes a selfish trait that can undermine colony

337 reproductive interests. To prevent selfish reproduction among workers, social insects have

338 evolved anti-cheater strategies, where colony members will systematically destroy eggs laid by

339 workers (Ratnieks and Visscher 1989). Tumor suppressor genes of multi-cellular organisms 
340 perform a similar function by recognizing and destroying cells that violate normal growth

341 regulation and thereby preventing outgrowths of genetically selfish cancer cells (Nunney 1999).

342 Finally, group selection dynamics can even result in Simpson's paradox, in which the overall

343 frequency of cooperators increases despite their systematic tendency to decrease within groups

344 (Chuang, et al. 2009). The fact that a trait can spread even as it selects against in every individual

345 carrier shows the potential for selection on long-term growth rate to prevail over selection on

346 individuals.

\section{Limitations of fitness averages}

Limitations due to short-sighted selection

352 A central theme in many of the treatments of lineage-variable fitness effects is that fitness

353 differences can be averaged across a lineage using concepts like geometric mean fitness and

354 inclusive fitness. These extended fitness averages provide a convenient way to determine if an

355 allele has a positive or negative affect on a lineage- by instead determining whether it increases

356 long-term the rate of spread of a lineage as a whole. We also note the equivalency between these

357 concepts and several related averages that depict long-term growth rates. For example, pathogens

358 are widely assumed to maximize their long-term transmission success, $R_{0}$ (Alizon, et al. 2009,

359 Anderson and May 1982). Similarly, Lyapunov exponents are sometimes used to derive long-

360 term growth rates in variable environments (Kussell and Leibler 2005) and the concept of

361 invasion fitness in evolutionary game theory (Sidebar 3) indicates whether natural selection tends

362 to favor a trait under frequency dependence (Lehmann, et al. 2016). Similar averages have been 
used to deal with variation in an allele's genetic background (Falconer 1994, Livnat and

364 Papadimitriou 2016). In general, averages across the variability in reproductive success are

365 meant to allow one to directly define an "effective" selection coefficient in order to identify

366 which allele increases fitness. An even more ambitious goal would be to salvage Equation 1, as

367 is the case under scenarios of rapid environmental change (Cvijović, et al. 2015).

369 Unfortunately, there are fundamental problems with the use of these averages that can preclude

370 natural selection from maximizing fitness averages. Specifically, shortsighted selection can drive

371 alleles to extinction, regardless of their long-term benefit to a lineage. This is most readily seen

372 in the case of a changing environment (Figure 2), where it has been noted in several contexts

373 (Cvijović, et al. 2015, Gerland and Hwa 2009, King and Masel 2007, Masel, et al. 2007).

374 Assume that a mutation arises in an environment in which it is beneficial and that the

375 environment is constant for $\tau$ generations. Provided it survives genetic drift, the allele will

376 increase in frequency following a logistic function and reach a frequency of one in

377 approximately $2 \cdot \ln (N s) / s$ generations (Desai and Fisher 2007). Thus, if $\tau \gg 2 \cdot \ln (N s) / s$, then

378 alleles will tend to arise and fix all in the same environment (Cvijović, et al. 2015). This provides

379 a straightforward threshold, beyond which natural selection is blind to the allele's long-term

380 benefit. Of course, this threshold is derived under the assumption of a well-mixed population of

381 constant size, and other factors such as demographic changes and population subdivision could

382 substantially extend this upper bound. Still, these considerations demonstrate an inherent time-

383 constraint imposed by evolution in finite populations, which only disappear as a mathematical

384 artifact in infinite populations (Figure 2C). 
386 Similar limitations can be seen whenever the timescale of change in the fitness effects of an

387 allele are greater than the time needed for natural selection to fix alleles conferring a short-term

388 advantage. For example, models of multi-level selection become dominated by shortsighted

389 selection of selfish phenotypes whenever group-level reproductive events are rare (Luo 2014).

390 This breakdown in favor of shortsighted selection is analogous to that in variable environments

391 (compare Figures 2B and 2D) and can be understood by considering the relative effects of

392 individual and group selection on changes to allele frequency. Natural selection takes about $s$

393 generations to double the frequency of a selfish trait within groups, where $s$ denotes the within-

394 group benefit of a selfish trait. On the other hand, increased rates of group reproduction in groups

395 of non-selfish individuals will double the frequency of a cooperative trait after approximately $w \cdot r$

396 generations, where $r$ is the group-level selection coefficient and $w$ is the number of individual

397 generations between group reproductive events. This heuristic reasoning implies that

398 shortsighted selection in favor of a selfish trait will dominate allele frequency changes and

399 preclude the evolution of cooperation whenever $s \gg w \cdot r$, which very closely matches results

400 derived by formal analysis (Luo 2014).

401

402

Beyond fitness averages

404 In addition to the role of extinction in tipping the outcome of selection toward shortsighted traits,

405 studies explicitly modeling variability across a lineage have yielded a number of additional

406 results that are not readily captured by Equation 1. Recently, Cvijović, et al. (2015) examined the

407 case of a periodic environment that alternates between two states. An allele that is favored in one

408 environment but disfavored in the next can follow unintuitive dynamics, particularly when large 
changes in allele frequency occur within environmental epochs. In the classic, lineage-invariant scenario discussed above, fixation of a neutral allele from a single starting copy requires

411 traversing from a starting frequency of $1 / N$ to a frequency of 1 by the action of genetic drift

412 alone. In contrast, mutations in a fluctuating environment experience selective pressures

413 continually, albeit of varying signs and intensities. This means that alleles can be driven to very

414 high or very low frequencies by natural selection and then achieve fixation or loss due to genetic

415 drift with far greater probability than predicted by Equation 1. This effect can cause the fixation

416 probability of an allele to increase well beyond the neutral expectation of $1 / N$, even when alleles

417 are neutral or deleterious on average. Furthermore, natural selection becomes less efficient at

418 recognizing long-term fitness effects- causing mutations to behave as though they were

419 effectively neutral, even when they are beneficial or deleterious on average. Finally, as

420 populations become smaller or swings in frequency more dramatic, fixation can become

421 independent of the average selection coefficient, creating conditions where the fixation

422 probability is not even a monotonically increasing function of long-term fitness.

424 Another intriguing result emerges when the mean reproductive success across a lineage is held

425 constant but its variance is altered. For example, Gillespie (1974) considered a model meant to

426 capture spatial variation in the environment by relaxing the assumption of a Poisson-distributed

427 number of offspring. Gillespie found that the natural way to quantify fitness is $w=\mu-\frac{1}{N} \sigma^{2}$

428 where $\mu$ is the mean number of offspring, $\sigma^{2}$ is its variance, and $N$ is the population size. A

429 striking feature of this model is the appearance of population size in the definition of fitness,

430 which suggests that the same allele can be favored or disfavored depending solely on the

431 population size. This same sort of dependence on population size arises in a model of fluctuating 
432 environments (Takahata, et al. 1975), as well as in mutators (André and Godelle 2006, Raynes, et

433 al. 2014, Wylie, et al. 2009). We emphasize that the population size dependence in the above

434 examples is distinct from that of Equation 1, where population size influences the efficiency of

435 natural selection but does affect its sign. Instead, variability in fitness across a lineage makes it

436 possible that a subset of individuals will experience strong selective pressures that are not

437 dominated by drift, even in small populations. This implies that genetic drift and natural

438 selection do not, in general, scale according the relationship in Equation 1.

440 Perhaps the most intriguing feature of lineage variability is the possibility that the fate of an

441 allele may not always be reducible to a selection coefficient at all. This is certainly the case for

442 the evolution of mutation rate modifiers, where the succession of de novo beneficial and

443 deleterious mutations results not only in variability in the distribution of offspring numbers

444 across a lineage, but also in temporal autocorrelation in this distribution among the resulting sub-

445 lineages (Figure 2C). Consequently, the offspring distribution is not only changing through time,

446 but is also inherently linked to the underlying lineage dynamics. This implies that one is unable

447 to define any selection coefficient for a mutator that predicts $P_{\text {fix. }}$, but must instead derive $P_{\text {fix }}$

448 directly under models that explicitly capture the dynamics of secondary mutations and clonal

449 interference (Good and Desai 2016). Although one could then use $P_{\text {fix }}$ to retrospectively define

450 an effective coefficient for any given population size using Equation 1 (Wylie, et al. 2009), it

451 seems that one cannot generally define such a selection coefficient a priori. Moreover, even

452 given such an effective selection coefficient, true $P_{\text {fix }}$ doesn't scale with $N$ in the manner

453 predicted by Equation 1 (Good and Desai 2016). It remains to be seen whether a similar inability 
454 to reduce lineage fate to any effective selection coefficient might emerge in the context of

455 variable environments and other examples of lineage-variable fitness effects.

\section{Implications for infectious disease evolution}

459 One of the most promising applications of models considering lineage-variable fitness effects is

460 in predicting and controlling the evolution of infectious diseases. Medically important traits such

461 as pathogen virulence and drug resistance evolve rapidly and there has been considerable interest

462 in the development of evolution-proof vaccines and antibiotics (Allen, et al. 2014, Day and Read

463 2016, Huijben, et al. 2013, Read, et al. 2011). Pathogen lineages experience a variety of extrinsic

464 environmental changes including a dynamic immune response, a diverse set of tissues and hosts,

465 and varying exposure to drugs. Additionally, since reproduction occurs both within and between

466 hosts, multi-level selection can create conflicting selective pressures operating over different

467 timescales (Kawashima, et al. 2009, Levin and Bull 1994). Finally, the dynamic immune

468 response targeting antigenic epitopes has resulted in the selective pressures favoring mutator

469 genes capable of immune evasion and antigenic evolvability (Deitsch, et al. 2009, Graves, et al.

470 2013, Moxon, et al. 1994). Variability across lineages therefore appears to be the rule rather than

471 the exception in infectious disease evolution.

472

473 Predicting pathogen evolution and designing evolution-proof drugs will be greatly aided by

474 models that combine the various selective pressures operating at different levels and timescales

475 during the pathogen life-cycle. Traditional models have generally assumed that natural selection

476 will favor traits that increase the long-term epidemiological success. For example, virulence is 
477 widely regarded as an adaptation to balance the increased rate of transmission by more

478 aggressive diseases with the reduced duration of infection caused by host mortality or immune

479 selection (Alizon, et al. 2009, Alizon and Michalakis 2015, Anderson and May 1982, Bull and

480 Lauring 2014). However, the assumption that natural selection will maximize transmission

481 success is analogous to selection maximizing other long-term measures of lineage success, like

482 geometric mean fitness, and is therefore sensitive to the limitations discussed above (Figure 2).

483 Specifically, shortsighted selection occurring within-hosts may act as a barrier for traits that

484 could increase long-term transmission success (Levin and Bull 1994; Sidebar 2). Indeed, models

485 that include mutation or competition between strains within-hosts or other ecological dynamics

486 have demonstrated the inability of selection to maximize transmission success (Alizon, et al.

487 2013, Bonhoeffer and Nowak 1994, Day 2003).

489 There is broad support for the prediction that shortsighted selection and selection acting to 490 increase traits that are beneficial on average can interact to shape infectious disease traits. For 491 example, empirical studies in HIV (Alizon and Fraser 2013) and enteric bacteria (Giraud, et al.

492 2001) show how short-sighted selection can dominate patterns of evolution and lead to

493 reductions in long-term transmission success. In Salmonella enterica, the need to maintain costly

494 virulence factors that are susceptible to short-sighted selection for cheaters appears to have

495 favored a strategy of cheater prevention that help to stabilize long-term infectivity (Diard, et al.

496 2013, Frank 2013, Mulder and Coombes 2013). Further theoretical progress on the role of

497 interaction between the differing timescales of selection in pathogens could come from models

498 that explicitly combine mechanistic within-host processes with long-term epidemiological

499 dynamics (Coombs, et al. 2007, Day and Gandon 2007, Gilchrist and Coombs 2006, Mideo, et 
500 al. 2008). In addition, new experimental technologies such as lineage tracking of pathogens using

501 barcode deep-sequencing (Blundell and Levy 2014, Levy, et al. 2015) offer exciting

502 opportunities to measure selective pressures occurring within-hosts and integrate them with more

503 traditional epidemiological data.

\section{Conclusions}

506

507 Despite historical emphasis on individual fitness effects shaping the fate of an allele, such a

508 measure of fitness cannot always capture long-term evolutionary behavior when variability in

509 fitness effects arise due to environmental, genetic or social interactions (Table 1, Figure 1). In

510 some cases, averaging lineage-variable fitness across the various environmental, genetic, and

511 social contexts an allele encounters allows for the application of classical population genetics

512 results based on traditional notions of fitness related to individual survival and reproduction.

513 However, this approach can fail in finite populations where an allele's predicted fate can be

514 interrupted by fixation or extinction due to shortsighted selection (Figure 2B). Furthermore,

515 genetic drift and natural selection interact in unexpected ways when variability in fitness effects

516 occurs over a comparable timescale to allele frequency (Cvijović et al. 2015, Figure 2D). More

517 strikingly, examples from studies of mutation rate modifiers indicate that there may be no way to

518 summarize the direction of natural selection on an allele without simply modeling its long-term

519 lineage dynamics (Good and Desai 2016). Taken together, these findings may have particular

520 relevance for the study of infectious pathogens, where alleles are likely to experience variability

521 due to a combination of environmental, genetic, and social interactions. 
523 Variability in the fitness effects of an allele challenge the conventional premise of population

524 genetics that individual offspring number can be drawn from a fixed distribution for all members

525 of a lineage (Figure 1). Cases where the typical assumption of a Poisson offspring distribution

526 have been relaxed (Gillespie 1974) have yielded intriguing new evolutionary properties such as

527 dependence on both the mean and variance in fitness effects and a critical effect of population

528 size in determining whether an allele is beneficial. Other examples allow properties of the

529 offspring distribution to vary in time, but still assume that the form of the distribution is fixed

530 (Cvijović, et al. 2015). In yet other cases, it appears that allele frequency dynamics cannot

531 always be reduced to one of independent draws from any offspring distribution, time-dependent

532 or not. This effect is most recognizable in mutators, where the offspring distribution changes in a

533 manner that is inseparable from the underlying lineage dynamics caused by secondary mutations

534 and selection on sub-lineages (Figure 1C). Thus, while theoretical progress has been made in

535 understanding processes where the offspring distribution takes on more general forms (Cannings

536 1974, Der, et al. 2011), we are still far from a population genetics theory with which to predict

537 the fate of an allele in general scenarios of lineage-variable fitness effects.

538

539 Lineage variability also highlights the need for caution when interpreting the adaptive

540 significance of biological traits in nature. Emphasis has often been placed on individual fitness

541 effects at the expense of neglecting the ability of selection to favor traits that have longer term

542 consequences on the fate of an allele (Williams 1966). Indeed, there are a plurality of definitions

543 of fitness (Orr 2009) with each generalizing the concept of fitness under a particular source of

544 lineage variability but none that appear sufficiently general to account for all examples. Caution

545 is warranted when considering traits in the context of their long-term effects on a lineage, since 
546 such traits are inherently susceptible to shortsighted selection (Figure 2). Thus, while it is often

547 safe to assume that selection will favor traits on the basis of extended fitness metrics, it is also

548 important to consider the inherent limitations in the ability of natural selection to optimize any

549 measure of fitness.

550

551 Acknowledgements

552

553 The authors gratefully acknowledge Erol Akçay, Michael Desai, Steven A. Frank, Joanna Masel,

554 Paul Rainey, Richard Watson, Eugene Raynes and other members of the Weinreich lab for

555 numerous constructive comments on an earlier draft. C.J.G. is supported in part by NSF

556 Graduate Research Fellowship 1644760, NSF 1501355 and NSF DGE 0966060. D.M.W.

557 supported in part by NSF DEB-1556300 and NIH R01GM095728. 


\section{Literature cited}

561

562 Akçay E, Van Cleve J. 2016. There is no fitness but fitness, and the lineage is its bearer. Philos. Trans. R. Soc. Lond., B, Biol. Sci. 371: 20150085

Alizon S, de Roode JC, Michalakis Y. 2013. Multiple infections and the evolution of virulence. Ecol. Lett. 16: 556-67

Alizon S, Fraser C. 2013. Within-host and between-host evolutionary rates across the HIV-1 genome. Retrovirology 10: 49 hypothesis: history, current state of affairs and the future. J. Evol. Biol. 22: 245-59

573

574 Alizon S, Michalakis Y. 2015. Adaptive virulence evolution: the good old fitness-based approach. Trends Ecol. Evol. 30: 248-54

577 Allen RC, Popat R, Diggle SP, Brown SP. 2014. Targeting virulence: can we make evolutionproof drugs? Nature Rev. Microbiol. 12: 300-08 
583 André J-B, Godelle B. 2006. The Evolution of Mutation Rate in Finite Asexual Populations. Genetics 172: 611-26

Balaban NQ, Merrin J, Chait R, Kowalik L, Leibler S. 2004. Bacterial persistence as a phenotypic switch. Science 305: 1622-5

Blundell JR, Levy SF. 2014. Beyond genome sequencing: Lineage tracking with barcodes to study the dynamics of evolution, infection, and cancer. Genomics 104: 417-30

Bonhoeffer S, Nowak MA. 1994. Mutation and the Evolution of Virulence. Proc. R. Soc. B 258:

Bull JJ, Lauring AS. 2014. Theory and empiricism in virulence evolution. PLoS Pathog. 10: e1004387

Cannings C. 1974. The latent roots of certain Markov chains arising in genetics: a new approach,

604 Childs DZ, Metcalf C, Rees M. 2010. Evolutionary bet-hedging in the real world: empirical evidence and challenges revealed by plants. Proc. R. Soc. B 277: 3055-64 
607 Chuang JS, Rivoire O, Leibler S. 2009. Simpson's Paradox in a Synthetic Microbial System.

608 Science 323: 272-75

609

610 Clauss M, Venable D. 2000. Seed germination in desert annuals: an empirical test of adaptive bet hedging. Am. Nat. 155: 168-86

612

613 Colegrave N. 2002. Sex releases the speed limit on evolution. Nature 420: 664-66

614

615 Coombs D, Gilchrist MA, Ball CL. 2007. Evaluating the importance of within- and between-host selection pressures on the evolution of chronic pathogens. Theor. Popul. Biol. 72: 576-91

617

Cooper TF. 2007. Recombination speeds adaptation by reducing competition between beneficial mutations in populations of Escherichia coli. PLoS biology 5: e225

620

621 Costanzo M, VanderSluis B, Koch EN, Baryshnikova A, Pons C, et al. 2016. A global genetic interaction network maps a wiring diagram of cellular function. Science 353: aaf1420

623

624 Cvijović I, Good BH, Jerison ER, Desai MM. 2015. Fate of a mutation in a fluctuating

625 environment. Proc. Natl. Acad. Sci. USA 112: E5021-E28

626

627 Day T. 2003. Virulence evolution and the timing of disease life-history events. Trends Ecol. Evol. 18: 113-18 
630 Day T, Gandon S. 2007. Applying population-genetic models in theoretical evolutionary epidemiology. Ecol. Lett. 10: 876-88

632

633 Day T, Read AF. 2016. Does high-dose antimicrobial chemotherapy prevent the evolution of resistance? PLoS Comput. Biol. 12: e1004689

635

Deitsch KW, Lukehart SA, Stringer JR. 2009. Common strategies for antigenic variation by bacterial, fungal and protozoan pathogens. Nature Rev. Microbiol. 7: 493-503

638

639 Dempster ER. 1955. Maintenance of genetic heterogeneity. Cold Spring Harb. Symp. Quant. Biol. 20: 25-31

642 Denamur E, Matic I. 2006. Evolution of mutation rates in bacteria. Mol. Microbiol. 60: 820-27

644 Der R, Epstein CL, Plotkin JB. 2011. Generalized population models and the nature of genetic 645 drift. Theor. Popul. Biol. 80: 80-99

646

647 Desai MM, Fisher DS. 2007. Beneficial mutation selection balance and the effect of linkage on positive selection. Genetics 176: 1759-98

649 cooperative virulence by the expression of an avirulent phenotype. Nature 494: 353-56 
653 Eshel I. 1973. Clone-Selection and Optimal Rates of Mutation. J Appl. Probab. 10: 728-38

654

655 Eshel I, Feldman MW. 1970. On the evolutionary effect of recombination. Theor. Popul. Biol. 1:

$88-100$

657

658 Eshel I, Feldman MW, Bergman A. 1998. Long-term Evolution, Short-term Evolution, and Population Genetic Theory. Journal of Theoretical Biology 191: 391-96

660

Falconer DS. 1994. Introduction to Quantitative Genetics. Essex, England: Longman Scientific and Technical. $438 \mathrm{pp}$.

663

664 Frank SA. 1995. Mutual policing and repression of competition in the evolution of cooperative groups. Nature 377: 520-22

666

667 Frank SA. 2013. Microbial Evolution: Regulatory Design Prevents Cancer-like Overgrowths.

670 Frank SA, Slatkin M. 1990. Evolution in a variable environment. Am. Nat. 136: 244-60

671

672 Fraser D, Kaern M. 2009. A chance at survival: gene expression noise and phenotypic diversification strategies. Mol Microbiol 71: 1333-40 
675 Gerland U, Hwa T. 2009. Evolutionary selection between alternative modes of gene regulation.

Proc. Natl. Acad. Sci. USA 106: 8841-46

677

678 Gerrish PJ, Lenski RE. 1998. The fate of competing beneficial mutations in an asexual population. Genetica 102: 127

680

681 Gilchrist MA, Coombs D. 2006. Evolution of virulence: interdependence, constraints, and selection using nested models. Theor. Popul. Biol. 69: 145-53

683

684 Gillespie JH. 1973. Natural selection with varying selection coefficients-a haploid model. Genet.

685

Res. 21: 115-20

686

687 Gillespie JH. 1974. Natural selection for within-generation variance in offspring number.

688 Genetics 76: 601-06

689

690 Giraud A, Matic I, Tenaillon O, Clara A, Radman M, et al. 2001. Costs and benefits of high mutation rates: adaptive evolution of bacteria in the mouse gut. Science 291: 2606-8

692

693 Goddard MR, Godfray HCJ, Burt A. 2005. Sex increases the efficacy of natural selection in experimental yeast populations. Nature 434: 636-40

695

696 Good BH, Desai MM. 2016. Evolution of Mutation Rates in Rapidly Adapting Asexual Populations. Genetics 204: 1249-66 
699 Gore J, Youk H, Van Oudenaarden A. 2009. Snowdrift game dynamics and facultative cheating

700 in yeast. Nature 459: 253-56

701

702 Graves CJ, Ros VI, Stevenson B, Sniegowski PD, Brisson D. 2013. Natural selection promotes antigenic evolvability. PLoS Pathog. 9: e1003766

704

705

Gremer JR, Venable DL. 2014. Bet hedging in desert winter annual plants: optimal germination 706 strategies in a variable environment. Ecol. Lett. 17: 380-7

707

708

Haigh J. 1978. The accumulation of deleterious genes in a population-Muller's Ratchet. Theor.

709 Popul. Biol. 14: 251-67

710

711 Haldane JBS. 1927. A mathematical theory of natural and artificial selection. Part V: Selection and mutation. Proc. Camb. Phil. Soc 23: 838-44

713

714 Hamilton WD. 1964. The genetical evolution of social behaviour. I. Theor. Popul. Biol. 7: 1-16

715

716 Hamilton WD, Axelrod R, Tanese R. 1990. Sexual reproduction as an adaptation to resist parasites (a review). Proc. Natl. Acad. Sci. USA 87: 3566-73

719 Hopper KR. 1999. Risk-spreading and bet-hedging in insect population biology 1. Annu. Rev. 
722 Huijben S, Bell AS, Sim DG, Tomasello D, Mideo N, et al. 2013. Aggressive chemotherapy and the selection of drug resistant pathogens. PLoS Pathog 9: e1003578

724

725 Jones SE, Lennon JT. 2010. Dormancy contributes to the maintenance of microbial diversity. Proc Natl Acad Sci U S A 107: 5881-6

727

728 Kawashima Y, Pfafferott K, Frater J, Matthews P, Payne R, et al. 2009. Adaptation of HIV-1 to human leukocyte antigen class I. Nature 458: 641-45

731 Kimura M. 1962. On the probability of fixation of mutant genes in a population. Genetics 47:

734 King OD, Masel J. 2007. The evolution of bet-hedging adaptations to rare scenarios. Theor. Popul. Biol. 72: 560-75

736

737 Kondrashov AS. 1988. Deleterious mutations and the evolution of sex. Nature 336: 435-40

739 Koschwanez JH, Foster KR, Murray AW. 2013. Improved use of a public good selects for the evolution of undifferentiated multicellularity. eLife 2: e00367

742 Kryazhimskiy S, Rice DP, Jerison ER, Desai MM. 2014. Global epistasis makes adaptation predictable despite sequence-level stochasticity. Science 344: 1519-22 
745 Kussell E, Leibler S. 2005. Phenotypic Diversity, Population Growth, and Information in Fluctuating Environments. Science 309: 2075-78

748 Lang GI, Rice DP, Hickman MJ, Sodergren E, Weinstock GM, et al. 2013. Pervasive genetic hitchhiking and clonal interference in forty evolving yeast populations. Nature 500: 571-

750 4

751

752 LeClerc JE, Li B, Payne WL, Cebula TA. 1996. High mutation frequencies among Escherichia coli and Salmonella pathogens. Science 274: 1208

755 Lehmann L, Mullon C, Akçay E, Van Cleve J. 2016. Invasion fitness, inclusive fitness, and reproductive numbers in heterogeneous populations. Evolution 70: 1689-702

757

758 Levin BR, Bull JJ. 1994. Short-sighted evolution and the virulence of pathogenic

759 microorganisms. Trends in Microbiology 2: 76-81

760

761 Levins R. 1968. Evolution in changing environments: Some theoretical explorations. Princeton, 762 NJ: Princeton University Press. 120 pp.

763

764 Levy SF, Blundell JR, Venkataram S, Petrov DA, Fisher DS, Sherlock G. 2015. Quantitative evolutionary dynamics using high-resolution lineage tracking. Nature 519: 181-+ 
767 Levy SF, Ziv N, Siegal ML. 2012. Bet hedging in yeast by heterogeneous, age-correlated expression of a stress protectant. PLoS Biol. 10: e1001325

Lewontin RC, Cohen D. 1969. On population growth in a randomly varying environment. Proc. Natl. Acad. Sci. USA 62: 1056-60

772

773 Livnat A, Papadimitriou CH. 2016. Sex as an Algorithm: The Theory of Evolution Under the Lens of Computation. Commun. ACM 59: 84-93

775

776

Luo S. 2014. A unifying framework reveals key properties of multilevel selection. Theor. Popul.

Biol. 341: 41-52

778

779

780

Lynch M. 2007. The frailty of adaptive hypotheses for the origins of organismal complexity. Proc. Natl. Acad. Sci. USA 104: 8597-604

781

782 Masel J, King Oliver D, Maughan H. 2007. The Loss of Adaptive Plasticity during Long Periods of Environmental Stasis. Am. Nat. 169: 38-46

785 Matic I, Radman M, Taddei F, Picard B, Doit C, et al. 1997. Highly variable mutation rates in commensal and pathogenic Escherichia coli. Science 277: 1833-34 
790 Maynard Smith J. 1978. The evolution of sex: Cambridge Univ Press

791

792 Maynard Smith J. 1982. Evolution and the Theory of Games: Cambridge Univ Press

793

794 Maynard Smith J, Price G. 1973. The logic of animal conflict. Nature 246: 15

795

796 McDonald MJ, Rice DP, Desai MM. 2016. Sex speeds adaptation by altering the dynamics of molecular evolution. Nature 531: 233-36

798

799 Menu F, Roebuck J-P, Viala M. 2000. Bet-hedging diapause strategies in stochastic

800 environments. Am. Nat. 155: 724-34

801

802 Meyers LA, Bull JJ. 2002. Fighting change with change: adaptive variation in an uncertain world. Trends Ecol. Evol. 17: 551-57

805 Mideo N, Alizon S, Day T. 2008. Linking within- and between-host dynamics in the evolutionary epidemiology of infectious diseases. Trends Ecol .Evol. 23: 511-7

807

808 Morran LT, Schmidt OG, Gelarden IA, Parrish RC, Lively CM. 2011. Running with the Red Queen: host-parasite coevolution selects for biparental sex. Science 333: 216-18

810

811 Moxon ER, Rainey PB, Nowak MA, Lenski RE. 1994. Adaptive evolution of highly mutable loci in pathogenic bacteria. Curr. Biol. 4: 24-33 
814 Mulder DT, Coombes BK. 2013. Infection biology: Cheats never prosper. Nature 494: 321-22

815

816 Muller HJ. 1964. The relation of recombination to mutational advance. Mutat. Res. 106: 2-9

817

818 Nunney L. 1989. The Maintenance of Sex by Group Selection. Evolution 43: 245-57

819

820 Nunney L. 1999. Lineage selection and the evolution of multistage carcinogenesis. Proc. R. Soc.

821

B 266: 493-98

822

823 Nunney L. 1999. Lineage selection: natural selection for long-term benefit. In Levels of selection

Orr HA. 2009. Fitness and its role in evolutionary genetics. Nature Rev. Genet. 10: 531-9

827

828 Philippi T, Seger J. 1989. Hedging one's evolutionary bets, revisited. Trends Ecol. Evol. 4: 41-4

829

830 Phillips PC. 1998. The language of gene interaction. Genetics 149: 1167-71

831

832 Pigliucci M. 2008. Is evolvability evolvable? Nature Rev. Genet. 9: 75-82

833

834 Rainey PB, Rainey K. 2003. Evolution of cooperation and conflict in experimental bacterial

$835 \quad$ populations. Nature 425: $72-74$ 
837 Ratnieks FL, Visscher PK. 1989. Worker policing in the honeybee. Nature 342: 796-97

838

839 Raynes Y, Halstead AL, Sniegowski PD. 2014. The effect of population bottlenecks on mutation rate evolution in asexual populations. J. Evol. Biol. 27: 161-69

841

842 Read AF, Day T, Huijben S. 2011. The evolution of drug resistance and the curious orthodoxy of aggressive chemotherapy. Proc. Natl. Acad. Sci. USA 108: 10871-77

845 Simon B, Fletcher JA, Doebeli M. 2013. Towards a general theory of group selection. Evolution 67: $1561-72$

848 Sniegowski PD, Gerrish PJ, Johnson T, Shaver A. 2000. The evolution of mutation rates: separating causes from consequences. Bioessays 22: 1057-66 populations of E. coli. Nature 387: 703-05

853

854 Sniegowski PD, Murphy HA. 2006. Evolvability. Curr. Biol. 16: R831-R34

855

856 Stearns SC. 2000. Daniel Bernoulli (1738): evolution and economics under risk. J. Biosci. 25:

857 $221-8$ 
859 Szathmary E, Maynard Smith J. 1995. The major evolutionary transitions. Nature 374: 227-32

860

861 Takahata N, Ishii K, Matsuda H. 1975. Effect of temporal fluctuation of selection coefficient on gene frequency in a population. Proc. Natl. Acad. Sci. USA 72: 4541-45

863

864 Traulsen A, Hauert C. 2009. Stochastic evolutionary game dynamics. In Reviews of nonlinear

865 dynamics and complexity, ed. HG Schuster, pp. 25-61. Weinheim, Germany: Wiley-VCH Verlag GmbH \& Co.

867

868 Traulsen A, Nowak MA. 2006. Evolution of cooperation by multilevel selection. Proc. Natl. Acad. Sci. USA 103: 10952-55

870

871 Travisano M, Velicer GJ. 2004. Strategies of microbial cheater control. Trends Microbiol. 12:

872 $72-78$

873

874 Turner PE, Chao L. 1999. Prisoner's dilemma in an RNA virus. Nature 398: 441-43

875

876 Van Valen L. 1973. A new evolutionary law. Evolutionary theory 1: 1-30

877

878 Velicer GJ, Kroos L, Lenski RE. 2000. Developmental cheating in the social bacterium

879 Myxococcus xanthus. Nature 404: 598-601 
881 Via S, Gomulkiewicz R, De Jong G, Scheiner SM, Schlichting CD, Van Tienderen PH. 1995.

882

Adaptive phenotypic plasticity: consensus and controversy. Trends Ecol. Evol. 10: 212-7

883

884 Wagner GP, Altenberg L. 1996. Perspective: Complex adaptations and the evolution of evolvability. Evolution 50: 967-76

886

887 Wang X, Fu AQ, McNerney ME, White KP. 2014. Widespread genetic epistasis among cancer genes. Nature communications 5

889

Watson RA, Szathmáry E. 2016. How Can Evolution Learn? Trends in Ecology \& Evolution 31: $147-57$

892

893 Weinreich DM, Lan Y, Wylie CS, Heckendorn RB. 2013. Should Evolutionary Geneticists Worry about High Order Epistasis? Current Opinion in Development and Genetics 23: 700-07

896

897 Williams GC. 1966. Adaptation and natural selection: A critique of some current evolutionary thought: Princeton university press

899

Wylie CS, Ghim CM, Kessler DA, Levine H. 2009. The fixation probability of rare mutators in finite asexual populations. Genetics 181: 1595-602

902 


\section{Sidebar 1 - What is a lineage? (Typeset near 'Introduction')}

905 We define a lineage as the full genealogy of descendent copies of an allele starting from the

906 original copy and ending at its long-term fate: extinction, fixation, or maintenance as a stable

907 polymorphism in the population. A traditional approach in population genetics has been to

908 describe the long-term evolutionary fate of a mutant allele influencing some biological trait

909 under the combined influence of evolutionary processes like mutation, genetic drift, migration,

910 and natural selection. Using analytical approaches from stochastic process theory, this work

911 seeks to calculate the probability that such an allele ultimately reaches a frequency of one, or

912 achieves fixation, in the population. This approach places emphasis not solely on the individual

913 reproductive process but also on the long-term fate of a genetic lineage carrying the mutation. It

914 therefore captures a much larger class of phenomena where fitness may not be directly affected

915 among individual carriers of an allele but the allele instead influences the statistical properties of

916 a lineage (Wylie, et al. 2009). Our focus will be primarily on lineage of asexual haploid lineages,

917 which are easier to analyze and depict. However, the approach and definition of a lineage given

918 here extends naturally to sexual diploid organisms. 
920 Sidebar 2 - Geometric mean fitness (Typeset near beginning of 'Lineage-Variable Fitness

921 Effects')

922 A widely appreciated result regarding adaptations to varying environments is the principle that

923 natural selection will favor traits based on their geometric mean fitness. When reproductive

924 success changes between generations, natural selection favors traits that increase the long-term

925 geometric mean fitness (GMF). Reflecting the multiplicative nature of reproduction, GMF is the

926 product of fitness in each generation, raised to the reciprocal of the number of generations.

927 Algebraically, GMF $=\left(\prod_{t=1}^{n} w_{t}\right)^{1 / n}$, where $w_{t}$ is the Wrightian fitness of a trait in generation $t$.

928 The same quantity can be expressed as a linear average over the natural log of this fitness value,

$929 \mathrm{GMF}=\exp \left[\frac{1}{n} \sum_{t=1}^{n} \ln \left(w_{t}\right)\right]$. In practice, approximations are used such as GMF $\approx \mu-\sigma^{2} / \mu$,

930 where $\mu$ is the arithmetic mean fitness and $\sigma^{2}$ is the variance in fitness. This formula explicates

931 the fact that natural selection favors increases in mean fitness, but also decreases in the variance

932 of fitness. This implies that natural selection can be risk averse, favoring alleles with lower

933 variance in fitness even at the expense of decreasing fitness on average. 
Sidebar 3 - Evolutionary game theory (Typeset near end of 'Lineage-Variable Fitness Effects'or

beginning of 'Limitations of Fitness averages')

938 Evolutionary game theory (Maynard Smith 1982) analyzes an interaction among a set of

939 competing alleles or "strategies" and summarizes their effect in a matrix representing the fitness

940 payoff of all pairwise competitions among competitors. Such a framework is most useful in the

941 context of frequency dependent selection, where the fitness effects of an allele are not easily

942 summarized by a constant selection coefficient. Such a framework provides a natural way to

943 determine whether a new allele starting from a single copy will tend to increase in frequency or

944 "invade" a population that is fixed for an alternative allele. This leads to the concept of an

945 evolutionarily stable strategy or ESS, which is defined as a strategy or allele that cannot be

946 invaded by any alternative strategy starting at an initially small frequency. The ability of an allele

947 to invade, or invasion fitness, is a generalization of the notion of a selection coefficient to the

948 case of frequency dependent selection and describes the long-term stability of a mutation against

949 other competing mutations (Eshel, et al. 1998, Lehmann, et al. 2016). While there are notable

950 exceptions (Traulsen and Hauert 2009, Traulsen and Nowak 2006), game theoretic models are

951 typically deterministic and describe the central tendency for allele frequency change but not the

952 statistical properties of lineages in finite populations. 
953 Glossary of terms (To appear adjacent to first use of each term or phrase)

954

955 Lineage-variable fitness effects: Differing fitness effects of an allele between individuals due to

956 genetic, social, or environmental interactions.

957

958 Offspring distribution: A discrete probability distribution that captures the stochasticity in an

959 individual organism's reproductive success.

960

961 Cheater: An individual that benefits from cooperative interactions of other individuals without

962 itself contributing to the cost of cooperating.

963

964 Frequency dependent selection: A model in which the fitness of an allele depends on its

965 frequency in the population as a consequence of interactions between organisms.

966

967 Epistasis: The phenotypic effect of a mutation varies with genetic context.

968

969 Modifier loci: Loci responsible for genetic properties of a genome, such as mutation rate,

970 recombination rate and mutational robustness.

971

972 Indirect selection: Selection acting on a modifier locus mediated by genetic linkage with fitness

973 effects at other loci in the genome.

974 
975 Clonal interference: Competition between mutational independent asexual lineages, each

976 carrying one or more beneficial mutations.

977

978 Genetic drift: Stochastic variation in allele frequency as a consequence of stochasticity in

979 reproduction inherent in finite populations.

980

981 


\section{Figure Captions}

Figure 1. Variability in fitness across a lineage in diverse models. A large number of realistic

985 biological scenarios can result in the presence of variation in fitness across a lineage either

986 among contemporary individuals (vertical axis) or between individuals in time (horizontal axis).

987 Genealogies are shown for two competing allelic lineages indicated by circles. The focal lineage

988 is shaded yellow and the wild-type lineage is shaded black. Curves on the bottom of each panel

989 depict changes to the mean (blue) and variance (orange) of fitness across the focal lineage. A.

990 Lineage carrying a beneficial allele (yellow) rising to fixation under the classical scenario of

991 lineage invariance. B. Lineage carrying an allele that alternates from beneficial to deleterious in

992 a variable environment. Contemporary individuals share an identical fitness, and hence an

993 identical selection coefficient, but this quantity changes over time. C. Evolution of a mutator

994 lineage that experiences increased rates of both deleterious (red dots) and beneficial (grey

995 background) mutations. D. A cooperative lineage under a group selection model. Within-group

996 selective pressures cause the allele to be disfavored over short timescales. Groups with more

997 cooperative alleles tend to displace other groups over longer timescales (shown with solid grey

998 lines). In both $\mathbf{C}$ and $\mathbf{D}$, fitness in the lineage varies both among contemporary individuals and

999 over time. 
1001 Figure 2. Limitations of fitness averages in finite populations. Evolution in a periodic

1002 environment results in four distinct regimes characterized by the relative timescale of natural

1003 selection $(1 / s)$ and environmental change $(\tau)$. Simulation results of the model described by

1004 Cvijović et al. (2015) are shown in blue and the expected change of an allele with the same

1005 average fitness effect in a constant environment is shown in red. Unless otherwise noted,

1006 simulations are conducted with an average selection coefficient of 0.05 and a population size of

1007 100,000. Selection coefficients are held constant at \pm 0.06 within each environment while the

1008 timescale of environmental change is varied (beneficial environmental epochs are shaded grey

1009 while deleterious epochs are unshaded). A. When the environment changes fast relative to

1010 changes in allele frequency (small $s \tau)$, the average change in allele frequency is well

1011 approximated by a fitness average like geometric mean fitness. B. When the environment

1012 changes slower than the time of fixation of an allele (large $s \tau$ ), mutations tend to arise and fix all

1013 in the same environment, regardless of their average fitness effect. C. In infinite populations,

1014 averages like the geometric mean fitness are accurate regardless of the timescale of

1015 environmental change. This is an artifact of the fact that, in the absence of genetic drift, allele

1016 frequencies can become arbitrarily close to zero or one but never permanently achieve fixation or

1017 extinction. D. Average fitness breaks down when large fluctuations in allele frequency occur on

1018 a similar timescale to environmental change (intermediate $\mathrm{s} \tau$ ). This is due to the amplification of

1019 fluctuations by genetic drift whenever alleles reach very high or very low frequencies (Cvijović

1020 et al. 2015). Note that genetic drift occurring as the frequency of the allele approaches 1 causes it

1021 to respond only modestly to the second deleterious epoch. The allele subsequently achieved

1022 fixation much sooner than would be expected on the basis of its average fitness effect. 
1024 Table 1. Sources of variability across a lineage and associated adaptations (Typeset near

1025 'Introduction')

\section{Basis of variability in fitness}

\begin{tabular}{llll}
\cline { 2 - 3 } & Environmental & Genetic & Social \\
\hline Specific examples & Spatial variation & Sex & Kin selection \\
& Temporal variation & Mutation & Multi-level \\
& & & selection \\
& & Clonal interference & \\
\hline Adaptations & Bet-hedging & Sex/recombination & Cooperation \\
& & & \\
& & & \\
Phenotypic & Musticity & & Policing \\
& & &
\end{tabular}

Evolvability 
bioRxiv preprint doi: https://doi.org/10.1101/107847; this version posted March 30 2017. The copyright holder for this preprint (which was not certified by peer review) is the author/funder, who has granted bioRxiv a license to display the preprint in perpetuity. It is made available under aCC-BY-NC-ND 4.0 International license.

A

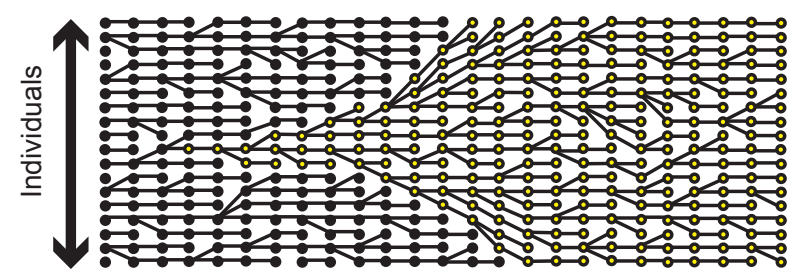

C
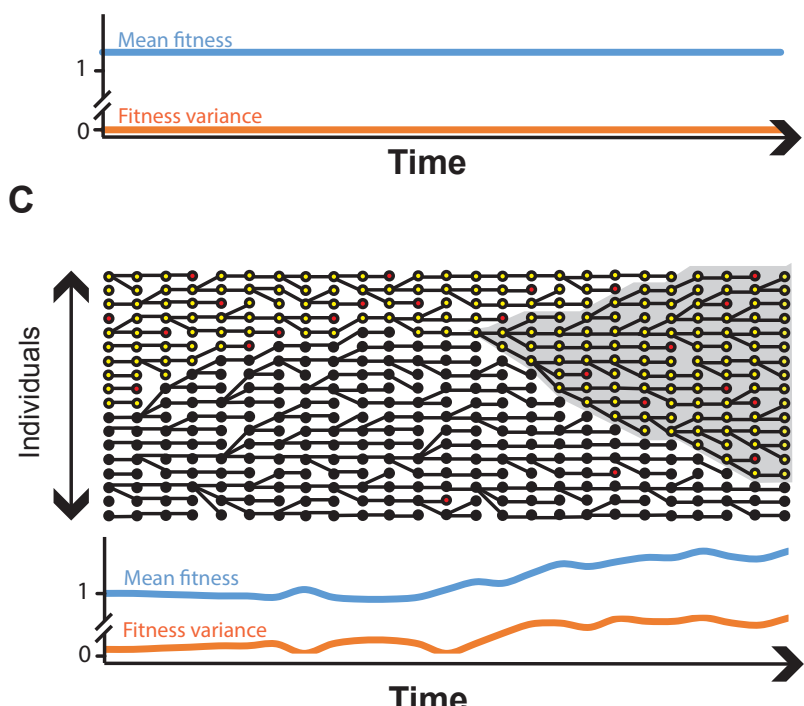

Figure 1
B
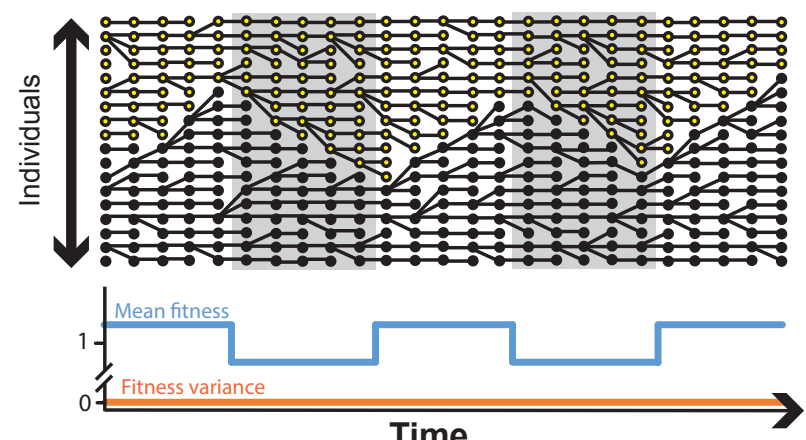

D

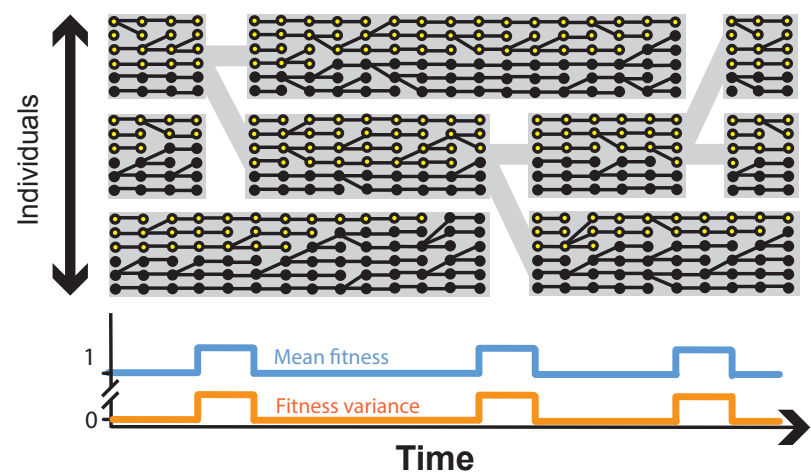

Time
A

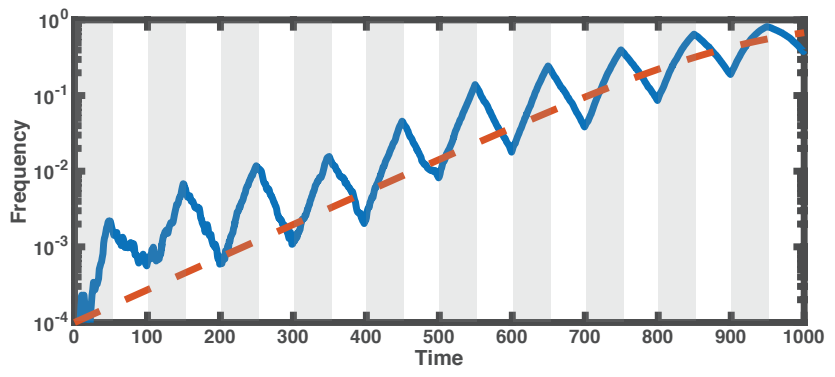

C

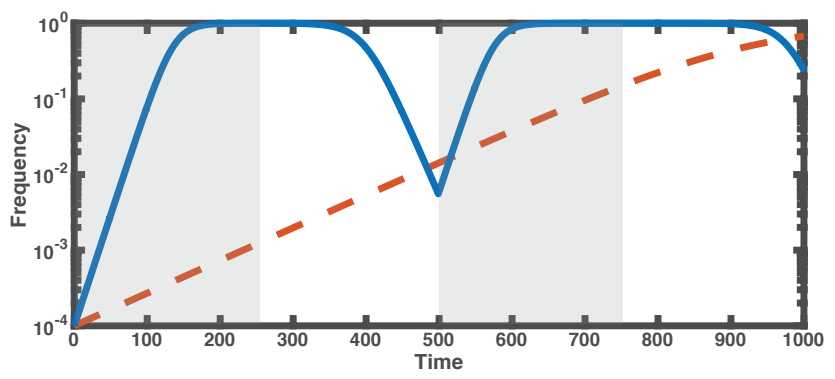

B

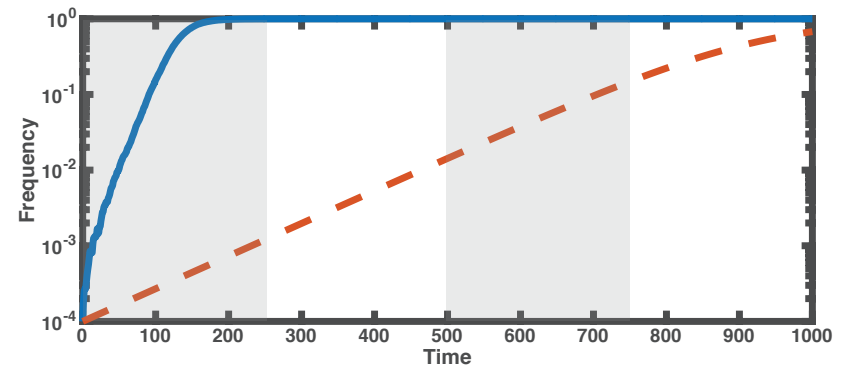

D

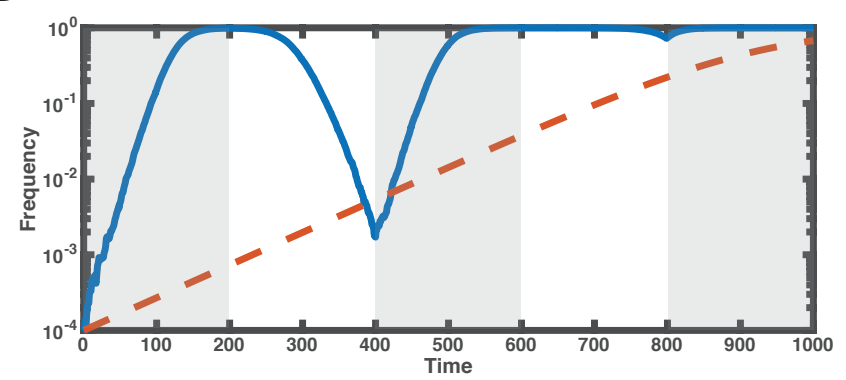

Figure 2 